Arq. Bras. Med. Vet. Zootec., v.67, n.1, p.227-234, 2015

\title{
Manejo pré-abate de ovelhas de descarte: perdas de peso corporal, qualidade da carne e comportamento animal
}

\author{
[Pre-slaughter management of discard ewes: body weight losses, meat \\ quality and animal behavior] \\ R.S.B. Pinheiro ${ }^{1}$, A.M. Jorge ${ }^{2}$, G.C. Miranda-de la Lama $^{3}$, H.B.A. Souza ${ }^{4}$ \\ ${ }^{1}$ Universidade Estadual Paulista "Júlio de Mesquita Filho" - FEIS - Ilha Solteira, SP \\ ${ }^{2}$ Universidade Estadual Paulista "Júlio de Mesquita Filho" - FMVZ - Botucatu, SP \\ ${ }^{3}$ Universidad Autónoma Metropolitana - UAM - Cidade do México, DF - México \\ ${ }^{4}$ Universidade Estadual Paulista "Júlio de Mesquita Filho" - FCAV - Jaboticabal, SP
}

\begin{abstract}
RESUMO
Objetivou-se com este estudo conhecer o comportamento de ovelhas da raça Santa Inês em diferentes estágios fisiológicos durante o manejo pré-abate, assim como a perda de peso corporal, valores de hematócrito do sangue das mesmas e a qualidade da carne. Utilizaram-se 21 ovelhas de descarte, que foram distribuídas nos respectivos tratamentos: $\mathrm{T} 1$ = ovelhas que permaneceram por 60 dias em lactação com seus respectivos cordeiros e abatidas um dia após o desmame dos mesmos; T2 = ovelhas que permaneceram por 60 dias em lactação com seus respectivos cordeiros e mais um período aproximado de 30 dias sem os cordeiros e posteriormente foram abatidas; e T3 = ovelhas que permaneceram por 60 dias em confinamento e que não pariram durante o ano. O peso das ovelhas após o transporte foi menor para as do tratamento 1 em comparação às do tratamento 2. Os valores de hematócrito no sangue das ovelhas antes do transporte, após o mesmo e após o jejum dos animais no curral de espera não diferiram entre si, com valor médio de 58,50\%. Concluiu-se que a qualidade da carne proveniente de ovelhas de descarte em diferentes estágios fisiológicos não é alterada pelo manejo pré-abate, quando realizado de maneira correta (evitando o estresse animal), quanto ao $\mathrm{pH}$ da carne.
\end{abstract}

Palavras-chave: ovinos, estresse animal, $\mathrm{pH}$ da carne, transporte rodoviário

\begin{abstract}
The aim of this study was to know the behavior of Santa Inês ewes in different physiological stages during the pre-slaughter management, as well as their body weight loss, blood hematocrit values and meat quality. 21 discard ewes were used, arranged into the following treatments: $T 1=$ ewes which remained in lactation for 60 days with their respective lambs and slaughtered one day after weaning; $T 2=$ ewes which remained in lactation for 60 days with their respective lambs and one more period of approximately 30 days without the lambs and afterwards slaughtered; and T3 = ewes which remained in confinement for 60 days and did not give birth during the year. The weight of ewes after transportation was lower for T1 in comparison with T2. Blood hematocrit values of ewes before and after transportation and after fast in the waiting pen were not different among the treatments, with mean value of 58.50\%. We concluded that the quality of meat of discard ewes in different physiological stages is not altered by preslaughter management, when correctly performed (avoiding animal stress), concerning meat $\mathrm{pH}$.
\end{abstract}

Keywords: sheep, animal stress, meat $p H$, road transportation

Recebido em 20 de abril de 2013

Aceito em 25 de junho de 2014

E-mail: rafaelsbp@bio.feis.unesp.br 


\section{INTRODUÇÃO}

De acordo com Costa (2002), os programas de qualidade de carne devem ter como ênfase mais do que a oferta de produtos seguros, nutritivos e saborosos; devem também ter compromissos com a produção sustentável e a promoção do bem-estar humano e animal, assegurando satisfação do consumidor e renda ao produtor, sem causar danos ao meio ambiente. Nesse cenário, o estudo do comportamento animal pode propiciar uma nova perspectiva para o modelo convencional de produção animal, trazendo alternativas a situações até agora não consideradas ou pouco compreendidas no bemestar animal.

O manejo pré-abate, com certeza, é uma das etapas de grande importância na produção animal, pois pode comprometer o resultado referente ao período de preparação dos animais para o abate (criação dos mesmos), podendo resultar em carcaças com problemas de qualidade (Costa et al., 2005).

O manejo pré-abate envolve uma série de situações não familiares para os animais de produção; no entanto, os mesmos, em algum momento, passarão por esse manejo para serem abatidos nos frigoríficos ou abatedouros legalizados. O manejo pré-abate inicia-se no embarque dos animais na propriedade e, no Brasil, o transporte rodoviário é o meio mais comum de condução dos animais de corte para o abate, independentemente da espécie animal. As operações de embarque e desembarque dos animais, se bem conduzidas, não produzem reações estressantes significativas (Kenny e Tarrant, 1987). Durante o transporte, os animais são privados de alimentos (sólido e líquido), o que causa perdas de peso corporal dos mesmos e, portando, percursos mais longos ou mais demorados promovem maiores perdas de peso corporal dos animais.

O período de descanso dos animais nos currais dos frigoríficos e abatedouros tem como objetivo principal reduzir o conteúdo do trato gastrointestinal para facilitar a evisceração, evitar contaminação da carcaça (Thornton, 1969) e, também, auxiliar o restabelecimento de parte das reservas de glicogênio muscular.
A carne DFD (Dark, Firm, Dry) é um problema causado pelo estresse crônico antes do abate do animal (Brown et al., 1990). Carnes DFD possuem valores de $\mathrm{pH}$ maiores que 5,80, apresentando uma vida de prateleira reduzida e a indesejável cor vermelha escura, sendo que a causa de pH elevado é a baixa concentração de glicogênio muscular no post-mortem (Immonen et al., 2000).

Não há bem definidas na literatura informações sobre o melhor estágio fisiológico para abater ovelhas de descarte, e nem há recomendação prática para se obter boa qualidade da carne proveniente desses animais (Pinheiro et al., 2012). Faltam dados sobre a influência do transporte desses animais na qualidade da carne. No entanto, tais informações são importantes, pois a ovinocultura de corte no Brasil está em franca expansão, além de muitos sistemas de produção estarem criando grandes quantidades de ovinos, gerando grande número de animais para descarte, principalmente ovelhas.

Diante do exposto, o objetivo deste estudo foi conhecer o comportamento de ovelhas da raça Santa Inês em diferentes estágios fisiológicos durante o manejo pré-abate, assim como da perda de peso corporal, os valores de hematócrito do sangue das mesmas e a qualidade da carne $(\mathrm{pH}$, temperatura e cor).

\section{MATERIAL E MÉTODOS}

Selecionaram-se para o presente experimento 21 ovelhas de descarte da raça Santa Inês (após um período de estação de monta) de um rebanho comercial localizado na cidade de Jaguariúna, São Paulo, Brasil. No início do estudo, os animais apresentaram peso corporal médio de aproximadamente $43 \pm 2,87 \mathrm{~kg}$, com idade média de $72 \pm 11$ meses. As fêmeas foram distribuídas nos seguintes tratamentos: $\mathrm{T} 1=$ ovelhas que permaneceram por 60 dias em lactação com seus respectivos cordeiros e foram abatidas um dia após o desmame dos mesmos; T2 = ovelhas que permaneceram por 60 dias em lactação com seus respectivos cordeiros e mais um período aproximado de 30 dias sem os cordeiros, no intuito de recuperar o peso corporal perdido durante $o$ período de amamentação e posteriormente foram abatidas; e T3 = ovelhas que permaneceram por 60 dias junto com as ovelhas do T1 e T2 até o abate e que não pariram 
durante o ano. Foram utilizadas sete ovelhas por tratamento.

As ovelhas permaneceram em regime de confinamento em uma instalação coberta, com área de solário, recebendo alimentação e água à vontade, durante todo o período do experimento. Todas as ovelhas foram abatidas no mesmo dia; portanto, as fêmeas do T2 pariram um mês antes das do T1, sendo que todos os partos foram simples (parição de um cordeiro por ovelha).

A dieta fornecida às ovelhas apresentou relação volumoso:concentrado de 70:30. O volumoso foi o feno de Tifton - 85 (Cynodon spp) e o concentrado era constituído por $69,6 \%$ de grão de milho moído, 24,8\% de farelo de algodão, $0,8 \%$ de calcário calcítico, $0,8 \%$ de fosfato bicálcico e $4 \%$ de suplemento mineral.

Os animais receberam duas refeições diárias, às 7 e às 16 horas, em cocho de madeira que permitiu $\mathrm{o}$ acesso de todos os animais ao mesmo tempo. Os cordeiros tiveram acesso a comedouro seletivo onde foi oferecido concentrado à vontade, no intuito de minimizar o desgaste promovido pelo período de lactação das ovelhas. Todos os animais tiveram acesso a sal comum fornecido em cocho. A composição bromatológica da dieta foi de $89,13 \%$ de matéria seca, $4,97 \%$ de matéria mineral, $11,81 \%$ de proteína bruta, $2,12 \%$ de extrato etéreo, $58,46 \%$ de fibra em detergente neutro e de $30,29 \%$ de fibra em detergente ácido.

Antes de conduzir as ovelhas da instalação de confinamento da propriedade para o caminhão, estas foram pesadas e também coletaram-se amostras de sangue da veia jugular das mesmas (coleta 1). Em seguida, as ovelhas foram embarcadas no caminhão e, após 10min do embarque, foram realizadas observações comportamentais dos animais por um período de 15min, com o caminhão parado e o motor desligado. Os parâmetros comportamentais avaliados foram: 1 - animal balindo ou não, 2 - animal deitado ou não, 3 - animal parado em pé ou não e 4 - animal movimentando normalmente (com tranquilidade) ou não.

Posteriormente, o caminhão foi ligado e iniciouse a viagem às 7:00 horas da manhã, saindo da cidade de Jaguariúna, São Paulo, até a cidade de Jaboticabal, São Paulo, chegando às 11:45 horas da manhã. O percurso de $296 \mathrm{~km}$ durou 4h45min em estrada asfaltada, em boas condições, sendo que, no meio da viagem, o caminhão parou por $15 \mathrm{~min}$ para realizar novamente as observações comportamentais dos animais. Antes do desembarque dos animais, em Jaboticabal, os mesmos permaneceram por $15 \mathrm{~min}$ no caminhão com o motor desligado, e nesse período foram observados novamente. O dia em que ocorreu o transporte dos animais estava ensolarado, com temperatura média de $26^{\circ} \mathrm{C}$ e umidade relativa do ar de 46\%. Após o desembarque, os animais permaneceram em curral de espera, onde foram pesados e coletadas amostras de sangue dos mesmos (coleta 2). Em seguida tiveram acesso à água.

Após um período de 10min no curral de espera, foi realizada a observação do comportamento das ovelhas por $15 \mathrm{~min}$ e repetida antes do abate dos animais.

As ovelhas permaneceram no curral de espera em jejum por 16 horas de dieta sólida antes do abate e, em seguida, foram pesadas e coletaram-se amostras de sangue (coleta 3) das mesmas. Posteriormente, os animais foram insensibilizados, quando então foram seccionadas as veias jugulares e as artérias carótidas para sangria. No momento da sangria, foi coletado sangue (coleta 4). Após a retirada da pele, a evisceração e a retirada das extremidades dos membros (patas) e da cabeça, as carcaças foram submetidas às medidas de $\mathrm{pH} 45$ minutos após abate (pH45mim) e temperatura por meio de um peagômetro digital portátil, introduzindo o eletrodo diretamente no músculo Longissimus lumborum, além da determinação da cor da carne por um colorímetro Minolta Chrome Meter CR300, operando no sistema CIE (L* luminosidade, $\mathrm{a}^{*}$ intensidade de vermelho e $\mathrm{b}^{*}$ intensidade de amarelo), calibrado para um padrão branco em ladrilho. Após isso, as carcaças foram transferidas para câmara frigorífica a $5^{\circ} \mathrm{C}$ por 24 horas e, posteriormente, determinou-se o $\mathrm{pH} 24$ horas (pH24h), a temperatura e a cor da carne.

O hematócrito foi determinado pela técnica do micro-hematócrito, a partir da coleta de amostras de sangue na veia jugular das ovelhas. Posteriormente, foi centrifugado e a leitura foi realizada com auxílio de escalas padronizadas do equipamento de micro-hematócrito. O resultado foi determinado em porcentagem. 
O delineamento experimental utilizado foi o inteiramente ao acaso com três tratamentos e sete repetições para os valores de $\mathrm{pH}$, temperatura e cor da carne. Para as análises de variância, utilizou-se o procedimento do Statistical Analysis System (SAS, 2002). As médias dos tratamentos foram comparadas pelo teste Tukey a $5 \%$ de probabilidade. As coletas de sangue (1, 2, 3 e 4) foram analisadas como medida repetida no tempo, pelo procedimento Mixed do software SAS (2002). As observações comportamentais foram todas anotadas durante o período préabate.

\section{RESULTADOS E DISCUSSÃO}

O peso corporal das ovelhas na propriedade, antes de serem embarcadas no caminhão, foi menor $(\mathrm{P}<0,05)$ para as do tratamento 1 (Tab. 1) em relação às dos tratamentos 2 e 3 . A explicação para isso está no fato de as ovelhas do tratamento 1 estarem ainda em lactação, o que acarreta grande mobilização de tecidos adiposos e até musculares.
O peso das ovelhas após o transporte (percurso de $296 \mathrm{~km}$ por 4 horas e 45 minutos) foi menor $(\mathrm{P}<0,05)$ para as do tratamento 1 em comparação com as do tratamento 2, e, quanto às do tratamento 3, não diferiram $(\mathrm{P}>0,05)$ dos demais tratamentos experimentais (Tab. 1). A perda de peso em quilos e em porcentagem do peso corporal das ovelhas durante o transporte não foi influenciada $(\mathrm{P}>0,05)$ pelos tratamentos experimentais, com valores médios de $2,28 \mathrm{~kg}$ e $5,15 \%$, respectivamente, estando de acordo com os valores relatados por Siqueira e Fernandes (1999), que obtiveram perda de peso, no transporte $(45 \mathrm{~km}$ do confinamento ao local do abate), de aproximadamente $4 \%$ do peso corporal em cordeiros que permaneceram em jejum de dieta hídrica antes do abate por 18 horas. A privação de alimento (sólido e líquido) conduz à perda de peso do animal (Roça, 2001). Portanto, a distância e o tempo da viagem estão altamente relacionados com a maior ou menor perda de peso dos animais, além das condições climáticas durante o transporte dos mesmos.

Tabela 1. Peso corporal de ovelhas de descarte durante o manejo pré-abate e perdas de peso corporal

\begin{tabular}{lccccc}
\hline \multirow{2}{*}{ Variável } & \multicolumn{3}{c}{ Tratamentos } & \multirow{2}{*}{ CV $(\%)$} & \multirow{2}{*}{ Teste F } \\
\cline { 2 - 4 } & T1 & T2 & T3 & & \\
\hline Peso corporal na propriedade $(\mathrm{kg})$ & $39,76 \mathrm{~b}$ & $46,82 \mathrm{a}$ & $46,90 \mathrm{a}$ & 12,14 & $4,04^{*}$ \\
Peso corporal após transporte $(\mathrm{kg})$ & $37,61 \mathrm{~b}$ & $44,8 \mathrm{a}$ & $44,20 \mathrm{ab}$ & 12,35 & $4,10^{*}$ \\
Perda de peso no transporte $(\mathrm{kg})$ & 2,14 & 2,01 & 2,70 & 18,06 & $3,07^{\mathrm{NS}}$ \\
Perda de peso no transporte $(\%)$ & 5,37 & 4,32 & 5,77 & 24,26 & $2,53^{\mathrm{NS}}$ \\
Peso corporal antes do abate $(\mathrm{kg})$ & $36,77 \mathrm{~b}$ & $44,36 \mathrm{a}$ & $43,34 \mathrm{ab}$ & 12,99 & $4,09^{*}$ \\
Perda de peso no jejum $(\mathrm{kg})$ & 2,10 & 1,93 & 1,86 & 17,37 & $3,01^{\mathrm{NS}}$ \\
Perda de peso no jejum (\%) & 5,40 & 4,18 & 4,11 & 23,05 & $0,63^{\mathrm{NS}}$ \\
Perda de peso total $(\mathrm{kg})^{1}$ & $2,98 \mathrm{ab}$ & $2,46 \mathrm{~b}$ & $3,55 \mathrm{a}$ & 28,08 & $4,16^{*}$ \\
Perda de peso total $(\%)^{1}$ & $7,51 \mathrm{a}$ & $5,37 \mathrm{~b}$ & $7,61 \mathrm{a}$ & 27,74 & $4,01^{*}$ \\
\hline
\end{tabular}

Letras distintas na mesma linha diferem significativamente entre si pelo teste Tukey a 5\% de probabilidade. NS = não significativo $(\mathrm{P}>0,05) . *$ significativo $(\mathrm{P}<0,05) .{ }^{1}$ Perda de peso no transporte + perda de peso no período de jejum.

O peso das ovelhas antes do abate foi inferior $(\mathrm{P}<0,05)$ para as do tratamento 1 em relação às do tratamento 2, e, quanto às do tratamento 3, não diferiram $(\mathrm{P}>0,05)$ dos demais tratamentos avaliados. O ocorrido é explicado pelo fato de as ovelhas em lactação (T1) já estarem anteriormente com um peso inferior ao das ovelhas que não estavam mais em lactação (T2), como demonstrado na Tabela 1.

A perda de peso das ovelhas durante o período em que permaneceram em jejum de sólidos no curral de espera, por aproximadamente 16 horas, antes do abate das mesmas, foi próximo $(\mathrm{P}>$ $0,05)$ entre os tratamentos experimentais, com valores médios de $1,96 \mathrm{~kg}$ e de 4,56\%, respectivamente. Apple et al. (1993) descreveram que ovinos com privação de alimentos por mais de 24 horas antes do abate apresentam maior estresse. Portanto, o tempo de jejum adotado neste estudo foi adequado para os animais, conforme resultados da Tabela 2.

A perda de peso total durante o manejo pré-abate das ovelhas diferiu $(\mathrm{P}<0,05)$ entre os tratamentos, com percentual menor para os animais do tratamento 2 em relação aos demais tratamentos experimentais, que foram próximos ( $>>0,05)$ entre si (Tab. 1). Jimenez Filho (2012) descreveu que a perda de peso em animais 
relatada na literatura varia de $0,75 \%$ a $11 \%$ do peso corporal nas primeiras 24 horas de privação de água e alimento. Portanto, o presente estudo (Tab. 1) apresentou valores normais de perda de peso, sendo essas perdas influenciadas por vários motivos, como tempo de transporte, condição climática e estágio fisiológico do animal.
Não houve interação $(\mathrm{P}>0,05)$ entre as coletas de sangue (1, 2, 3 e 4) durante o manejo pré-abate e o estágio fisiológico das ovelhas no valor de hematócrito (Tab. 2). Também não houve $(\mathrm{P}>0,05)$ diferença entre os tratamentos experimentais no valor de hematócrito.

Tabela 2. Valores de hematócrito de ovelhas em diferentes estágios fisiológicos durante o manejo préabate

\begin{tabular}{lcccc} 
& \multicolumn{3}{c}{ Tratamento (T) } & \multirow{2}{*}{ Média (M) } \\
\cline { 2 - 3 } Variável & T1 & T2 & T3 & \\
\hline Valor de hematócrito (\%) & & & & $57,33 \mathrm{~b}$ \\
\hline Antes do transporte (coleta 1) & 57,71 & 57,57 & 56,71 & $57,85 \mathrm{~b}$ \\
Após transporte (coleta 2) & 58,85 & 54,43 & 60,28 & $60,33 \mathrm{ab}$ \\
Após jejum (coleta 3) & 56,71 & 63,14 & 61,14 & $62,28 \mathrm{a}$ \\
Na sangria (coleta 4) & 59,85 & 66,00 & 61,00 & \\
Média (M) & 58,28 & 60,28 & 59,78 & \\
\hline
\end{tabular}

$\mathrm{CV} \%=10,30$

Teste $\mathrm{F}(\mathrm{T})=0,81 \mathrm{NS}$

Teste $\mathrm{F}(\mathrm{M})=2,95^{*}$

Teste $\mathrm{F}(\mathrm{T} \times \mathrm{M})=1,66 \mathrm{NS}$

Letras distintas na mesma coluna diferem significativamente entre si pelo teste Tukey a $5 \%$ de probabilidade. NS = não significativo $(\mathrm{P}>0,05) .{ }^{*}=$ significativo $(\mathrm{P}<0,05)$.

Os valores de hematócrito no sangue das ovelhas antes do transporte, após o mesmo e após o jejum dos animais no curral de espera não diferiram $(\mathrm{P}>0,05)$ entre si (Tab. 2). Na propriedade e após o transporte dos animais, o valor médio de hematócrito foi menor $(\mathrm{P}<0,05)$ que no momento da sangria dos animais (Tab. 2). De acordo com Güntler et al. (1986) e SchmidtNielsen (1996), a proporção do volume das células sanguíneas é dependente da solicitação física dos animais. Segundo os autores supracitados e Nunes et al. (2002), os animais sob esforço físico maior possuem valor de hematócrito mais elevado que aqueles sob menor esforço físico. Valores maiores de hematócrito podem indicar que o animal está em condições de estresse, como observado na Tabela 2 . Segundo Takahashi et al. (2006), o aumento do hematócrito e do número de células vermelhas pode ser reflexo da alta demanda do estresse. O aumento do hematócrito pode ser justificado pelo aumento relativo no número de hemácias, de acordo com o descrito no trabalho de Ferreira et al. (2009).

O pH da carne após 45 minutos do abate e 24 horas do mesmo não foi influenciado $(\mathrm{P}>0,05)$ pelos tratamentos experimentais, com valores médios de 6,40 e 5,52, respectivamente. Portanto, o manejo pré-abate não influenciou o $\mathrm{pH}$ da carne das ovelhas nem os estágios fisiológicos das mesmas.

Os valores de $\mathrm{pH}$ da carne, obtidos neste estudo (Tab. 3), são considerados normais para a espécie ovina e de carnes de qualidade (Sen et al., 2004; Pinheiro et al., 2010). De acordo com Devine et al. (1993) e Apple et al. (1995), cordeiros que passam por muito estresse antes do abate apresentam menor reserva de glicogênio muscular e valores de $\mathrm{pH}$ normalmente acima de 5,80, o que não ocorreu neste estudo (Tab. 3).

A maior influência do transporte na qualidade da carne é a diminuição do glicogênio muscular por atividade física ou estresse físico, promovendo uma queda anômala do $\mathrm{pH}$ post-mortem e originando a carne DFD (Roça, 2001). Essas condições estressantes geralmente são causadas pelo transporte prolongado (Knowles, 1999). De acordo com Jimenez Filho (2012), o transporte de animais, da fazenda ao abatedouro, apresenta alta correlação com rendimento de carcaça, $\mathrm{pH}$ e qualidade da carne produzida. 
Tabela 3. Valores médios de $\mathrm{pH}$, temperatura $\left({ }^{\circ} \mathrm{C}\right)$ e cor $\left(\mathrm{L}^{*}, \mathrm{a}^{*}\right.$ e $\left.\mathrm{b}^{*}\right)$ do músculo Longissimus lumborum proveniente de ovelhas abatidas em diferentes estágios fisiológicos

\begin{tabular}{|c|c|c|c|c|c|}
\hline \multirow{2}{*}{ Variável } & \multicolumn{3}{|c|}{ Tratamentos $(\mathrm{T})$} & \multirow{2}{*}{$\mathrm{CV}(\%)$} & \multirow{2}{*}{ Teste F } \\
\hline & $\mathrm{T} 1$ & $\mathrm{~T} 2$ & $\mathrm{~T} 3$ & & \\
\hline \multicolumn{6}{|l|}{$\mathrm{pH}$ da carne } \\
\hline 45 minutos após o abate & 6,32 & 6,45 & 6,44 & 2,02 & $2,23^{\mathrm{NS}}$ \\
\hline 24 horas após o abate & 5,55 & 5,53 & 5,49 & 1,27 & $1,48^{\mathrm{NS}}$ \\
\hline \multicolumn{6}{|l|}{ Temperatura da carne } \\
\hline 45 minutos após o abate & 36,04 & 36,66 & 35,91 & 5,74 & $0,26^{\mathrm{NS}}$ \\
\hline 24 horas após o abate & 7,51 & 6,53 & 6,64 & 15,84 & $0,28^{\mathrm{NS}}$ \\
\hline \multicolumn{6}{|l|}{ Cor da carne } \\
\hline $\mathrm{L}^{*} 45$ minutos após o abate & $32,68 \mathrm{a}$ & $30,72 b$ & $30,11 b$ & 4,48 & $6,44 * *$ \\
\hline L* 24 horas após o abate & $38,13 a$ & $35,53 \mathrm{ab}$ & $34,19 b$ & 5,98 & $6,05 * *$ \\
\hline$a^{*} 45$ minutos após o abate & 14,85 & 15,28 & 16,01 & 8,98 & $1,27^{\mathrm{NS}}$ \\
\hline $\mathrm{a}^{*} 24$ horas após o abate & 16,37 & 17,32 & 17,24 & 4,99 & $2,73^{\mathrm{NS}}$ \\
\hline$b^{*} 45$ minutos após o abate & 2,78 & 2,55 & 2,54 & 15,21 & $0,30^{\mathrm{NS}}$ \\
\hline b* 24 horas após o abate & 3,89 & 4,21 & 3,41 & 18,90 & $2,15^{\mathrm{NS}}$ \\
\hline
\end{tabular}

Letras distintas na mesma linha diferem significativamente entre si pelo teste Tukey a 5\% de probabilidade. NS = não significativo $(\mathrm{P}>0,05)$. $* *=$ significativo $(\mathrm{P}<0,01)$.

A temperatura do músculo Longissimus lumborum foi semelhante $(\mathrm{P}>0,05)$ entre os tratamentos avaliados, com valores médios de $36,20^{\circ} \mathrm{C}$ (temperatura 45 minutos após o abate) e de $6,89^{\circ} \mathrm{C}$ (temperatura 24 horas após o abate). Zeola et al. (2006), ao estudarem diferentes músculos da carcaça de cordeiros, não constataram diferenças entre os mesmos (Biceps femoris, Longissimus e Triceps brachii) para os valores de temperatura após abate e 24 horas do mesmo $\left(35,04^{\circ} \mathrm{C}\right.$ e $\left.4,15^{\circ} \mathrm{C}\right)$, sendo a temperatura 24 horas após abate inferior ao deste estudo (Tab. 3), provavelmente devido ao menor isolamento térmico e à quantidade de gordura da carcaça dos cordeiros.

As baixas temperaturas retardam o crescimento microbiano, e as reações químicas e enzimáticas que causam alterações na carne estão diretamente relacionadas à temperatura da carne. $\mathrm{O}$ valor da temperatura da carne após 24 horas do abate das ovelhas deste estudo (Tab. 3) estão de acordo com valores normais de resfriamento da carne de ruminantes (Jorge et al., 2006).

A luminosidade da carne apresentou diferença $(\mathrm{P}<0,01)$ entre os tratamentos experimentais (Tab. 3). As carnes provenientes das ovelhas do tratamento 2 e 3 tiveram valores menores de luminosidade do que as do tratamento 1 (Tab. 3) após 45 minutos do abate. A luminosidade da carne do tratamento1 foi superior à do tratamento 3 após 24 horas do abate. Os valores de vermelho e de amarelo do músculo Longissimus lumborum não foram influenciados $(\mathrm{P}>0,05)$ pelos tratamentos estudados nesta pesquisa (Tab. 3). Os valores da cor da carne obtidos neste estudo estão de acordo com valores normais da cor da carne ovina (Sañudo et al., 2000).

O comportamento das ovelhas não foi influenciado pelos diferentes momentos de observação (após o embarque no caminhão, no meio do trajeto da viagem e após a chegada ao destino) e estágios fisiológicos das ovelhas, com exceção de uma ovelha do tratamento 1 que balia a cada 2 min de cada período de observação dos animais dentro do caminhão. Todas as ovelhas permaneceram paradas em pé, durante todo tempo de observação no caminhão, independentemente do momento de observação. Segundo Batista et al. (1999), animais deitados durante o transporte podem ter um maior número de contusões. Portanto, pode gerar perda de qualidade da carcaça e de rendimento da mesma pela necessidade da retirada das lesões da carcaça, além de promover estresse aos animais.

O comportamento das ovelhas no curral de espera (após o desembarque) foi este: quatro ovelhas ficaram paradas em pé e as demais se movimentando normalmente, sendo que a ovelha que estava balindo no caminhão continuou a balir (uma vez por minuto durante o tempo de observação). Provavelmente os animais estavam tranquilos mesmo no manejo pré-abate, pois eram manejados diariamente na propriedade $\mathrm{e}$ porque eram do mesmo lote, não ocorrendo brigas entre as ovelhas. O comportamento das ovelhas antes do abate foi o seguinte: 10 ovelhas 
estavam deitadas e 11 paradas em pé, sendo que a mesma ovelha que estava balindo nos demais momentos observados anteriormente continuava a balir, porém com uma frequência menor (três vezes durante o período de observação). Essa ovelha que ficou balindo nos períodos observados foi a penúltima ovelha a parir do tratamento 1, o que explica tal comportamento, ou seja, o vínculo com o filhote e a habilidade materna.

Segundo Roça (2001), a tecnologia do abate de animais destinados ao consumo somente assumiu importância científica quando se observou que os eventos que se sucedem desde a propriedade rural até $o$ abate do animal tinham grande influência na qualidade da carne. Portanto, observar o comportamento dos animais é de extrema importância, pois pode evidenciar anormalidades que estejam ocorrendo com algum animal e que, consequentemente, possam influenciar o produto final. No Brasil, pouco se sabe, ainda, sobre as consequências de um manejo pré-abate inadequado, que, certamente, traz reflexos negativos na rentabilidade do pecuarista e do frigorífico (Silva et al., 2002). O manejo pré-abate vem sendo estudado em diferentes espécies animais (Santiago et al., 2012; Fernandes et al., 2013), pois pode apresentar alteração na qualidade da carne. Também há uma grande preocupação com o bem-estar animal no manejo pré-abate.

\section{CONCLUSÕES}

A porcentagem de perda de peso corporal durante o manejo pré-abate (transporte e período de permanência em jejum no curral) é superior para ovelhas que permaneceram em lactação recentemente em comparação à perda das ovelhas que já tinham desmamados os cordeiros. A qualidade da carne proveniente de ovelhas em diferentes estágios fisiológicos não é alterada pelo manejo pré-abate dos animais quanto ao $\mathrm{pH}$ da carne. Durante o manejo pré-abate, as ovelhas apresentam comportamento tranquilo quando é realizado de maneira correta (evitando o estresse animal), independentemente do estágio fisiológico em que se encontram, mesmo sendo uma situação desconhecida para os animais.

\section{REFERÊNCIAS}

APPLE, J.K.; DIKEMAN, M.E.; MINTON, J.E. et al. Effects of restrain and isolation stress and epidural blockade on endocrine and blood metabolite status, muscle glycogen metabolism, and indice of darck-cutting Longissimus muscle of Sheep. J. Anim. Sci., v.73, p.2295-2307, 1995.

APPLE, J.K.; UNRUH, J.A.; MINTON, J.E.; BARTLETT, J.L. Influence of repeated restraint and isolation stress and electrolyte administration on carcass quality and muscle electrolyte content of sheep. Meat Sci., v.35, p.191-203, 1993.

BATISTA, D.J.C.; SILVA, W.P.; SOARES, G.J.D. Efeito da distância de transporte de bovinos no metabolismo post-mortem. Rev. Bras. Agrocienc., v.5, p.152-156, 1999.

BROWN, S.N.; BEVIS, E.A.; WARRISS, P.D. A estimate of the incidence of dark cutting beef in the United Kingdon. Meat Sci., v.27, p.249258, 1990.

COSTA, M.J.R.P. Ambiência e qualidade de carne. In: CONGRESSO BRASILEIRO DAS RAÇAS ZEBUÍNAS, 5., 2002, Uberaba. Anais... Minas Gerais: ABCZ, 2002, p.170-174.

COSTA, O.A.D.; LUDKE, J.V.; COSTA, M.J.R.P. Aspectos econômicos e de bem estar animal no manejo dos suínos da granja até o abate. In: SEMINÁRIO INTERNACIONAL DE AVES E SUÍNOS, 4., 2005, Florianópolis. Anais... Santa Catarina: Avesui, 2005. p.1-25.

DEVINE, C.E.; GRAAFHUIS, A.E.; MUIR, P.D.; CHRYSTALL, B.B. The effect of growth rate and ultimate $\mathrm{pH}$ on meat quality of lamb. Meat Sci., v.35, p.63-77, 1993.

FERNANDES, J.I.M.; SAKAMOTO M.I.; PEITER, D.C. et al. Relação vitamina E: vitamina $\mathrm{C}$ sobre a qualidade da carne de frangos submetidos ao estresse pré-abate. Arq. Bras. Med. Vet. Zootec., v.65, p.294-300, 2013.

FERREIRA, F.; CAMPOS, W.E.; CARVALHO, A.U. et al. Parâmetros clínicos, hematológicos, bioquímicos e hormonais de bovinos submetidos ao estresse calórico. Arq. Bras. Med. Vet. Zootec., v.61, p.769-776, 2009.

GÜNTLER, H.; KETZ, A.; KOLB, E. Fisiologia veterinária. 2.ed. São Paulo: Guanabara. 1986. $569 \mathrm{p}$. 
IMMONEN, K.; KAUFFMAN, R.G.; SCHAEFER, D.M.; PUOLANNE E. Glycogen concentrations in bovine Longissimus dorsi muscle. Meat Sci., v.54, p.163-167, 2000.

JIMENEZ FILHO, D.L. Efeitos do transporte sobre a qualidade da carne - revisão. Med. Vet., v.6, p.26-31, 2012.

JORGE, A.M.; ANDRIGHETTO, C.; MILLEN, D.D. et al. Características bioquímicas da carne de bubalinos Mediterrâneo terminados em confinamento e abatidos em diferentes pesos. Cien. Rural, v.36, p.1534-1539, 2006.

KENNY, F.J.; TARRANT, P.V. The physiological and behavioural responses of crossbred Friesian steers to short-haul transport by road. Livest. Prod. Sci., v.17, p.63-75, 1987.

KNOWLES, T.G. A review of the road transport of cattle. Vet. Rec., v.144, p.197-201, 1999.

NUNES, A.S.; BARBOSA, O.R.; SAKAGUTI, E.S. et al. Efeito de dois regimes de suplementação alimentar e dois sistemas de produção, nos constituintes sangüíneos de cabras saanen durante a lactação. Rev. Bras. Zootec., v.31, p.1245-1250, 2002.

PINHEIRO, R.S.B.; JORGE, A.M.; SOUZA, H.B.A. Aceitação sensorial e composição centesimal da carne de ovelhas abatidas em diferentes estágios fisiológicos. Arq. Bras. Med. Vet. Zootec., v.64, p.1053-1059, 2012.

PINHEIRO, R.S.B.; JORGE, A.M.; SOUZA, H.B.A.; BOIAGO, M.M. Coloração da gordura e qualidade da carne de ovelhas de descarte abatidas em distintos estágios fisiológicos. Arq. Bras. Med. Vet. Zootec., v.62, p.468-474, 2010.

ROÇA, R.O. Abate humanitário: manejo antemortem. R. TeC Carnes, v.3, p.7-12, 2001.

SANTIAGO, J.C.; CALDARA, F.R.; SANTOS, V.M.O. et al. Incidência da carne PSE (pale, soft, exsudative) em suínos em razão do tempo de descanso pré-abate e sexo. Arq. Bras. Med. Vet. Zootec., v.64, p.1739-1746, 2012.
SAÑUDO, C.; ENSER, M.E.; CAMPO, M.M. et al. Fatty acid composition and sensory characteristics of lamb carcasses from Britain and Spain. Meat Sci., v.54, p.339-346, 2000.

SCHMIDT-NIELSEN, K. Fisiologia animal adaptação e meio ambiente. 5.ed. São Paulo: Santos, 1996. 546p.

SEN, A.R.; SANTRA, A.; KARIM, S.A. Carcass yield, composition and meat quality attributes of sheep and goat under semiarid conditions. Meat Sci., v.66, p.757-763, 2004.

SILVA, A.C.O.; BRAGATTO, S.A.; CLARO, C.A.C.; ANDRADE, A.A. Avaliação do transporte do rebanho bovino e seus reflexos na qualidade da carne e do couro brasileiro. In: ENCONTRO NACIONAL DE ENGENHARIA DE PRODUÇÃO. 22., 2002, Curitiba. Anais ... Paraná: ENEGEP, 2002. p.1-7.

SIQUEIRA, E.R.; FERNANDES, S. Pesos, rendimentos e perdas da carcaça de cordeiros Corriedale e mestiços Ile de France x Corriedale, terminados em confinamento. Cienc. Rural, v.29, p.143-148. 1999.

STATISTICAL Analysis System user's guide (2002) - SAS, Institute Inc. Version 9, 13 ed. São Paulo.

TAKAHASHI, L.S.; ABREU, J.S.; BILLER, J.D.; URBINATI, E.C. Efeito do ambiente pós-transporte na recuperação dos indicadores de estresse de pacus juvenis, Piaractus mesopotamicus. Acta Sci. Anim. Sci., v.28, p.469-475, 2006.

THORNTON, H. Compêndio de inspeção de carnes. Londres: Bailliere Tindall an Cassel, 1969. 665p.

ZEOLA, N.M.B.L.; SOUZA, P.A.; SOUZA, H.B.A. et al. Parâmetros de qualidade da carne de cordeiros submetida aos processos de maturação e injeção de cloreto de cálcio. Cien. Rural, v.36, p.1558-1564, 2006. 